\title{
The entropy solution of a hyperbolic-parabolic mixed type equation
}

\author{
Huashui Zhan*
}

*Correspondence:

Huashuizhan@163.com;

2012111007@xmut.edu.cn

School of Applied

Mathematics, Xiamen

University of Technology,

Xiamen 361024, China

\section{Abstract}

The entropy solution of the equation

$$
\frac{\partial u}{\partial t}=\Delta A(u)+\operatorname{div}(b(u)),(x, t) \in \Omega \times(0, T),
$$

is considered. Besides the usual initial value, only a partial boundary value is imposed. By choosing some special test functions, the stability of the solutions is obtained by Kruzkov's bi-variables method, provided that $\Omega \subset \mathbb{R}^{N}$ is an unit $n$-dimensional cube or the half space.

Keywords: Hyperbolic-parabolic mixed type equation, $n$-dimensional cube, Entropy solution, Kruzkov's bi-variables method

Mathematics Subject Classification: 35L65, 35K85, 35R35

\section{The boundary condition}

We consider the equation

$$
\frac{\partial u}{\partial t}=\Delta A(u)+\operatorname{div}(b(u)),(x, t) \in \Omega \times(0, T),
$$

and assume that

$$
A(u)=\int_{0}^{u} a(s) d s, \quad a(s) \geq 0, a(0)=0,
$$

where $\Omega \subset \mathbb{R}^{N}$ is a appropriately smooth open domain. Equation (1) is with hyperbolicparabolic mixed type, arises from the reaction diffusion problem (Wu et al. 2001), the stationary boundary layer theory (Oleinik and Samokhin 1999).

For Cauchy problem of Eq. (1), the paper (Vol'pert and Hudjaev 1967) was the first one to study its solvability, since then, many papers continued to dedicate to the problem, many excellent results were obtained, one can refer to Wu et al. (2001) and Refs. Bendahamane and Karlsen (2004), Brezis and Crandall (1979), Carrillo (1999), Chen and Dibenedetto (2001), Chen and Perthame (2003), Cockburn and Gripenberg (1999), Evans (1998), Karlsen and Risebro (2003), Kružkov (1970), Oleinik and Samokhin (1999), Vol'pert (1967), Vol'pert and Hudjaev (1967); Vol'pert and Hudjave (1975), Wu and Yin (1989), Wu et al. (2001), Zhan (2004), Zhao (1985), Zhao and Zhan (2005).

(c) 2016 The Author(s). This article is distributed under the terms of the Creative Commons Attribution 4.0 International License (http://creativecommons.org/licenses/by/4.0/), which permits unrestricted use, distribution, and reproduction in any medium, provided you give appropriate credit to the original author(s) and the source, provide a link to the Creative Commons license, and indicate if changes were made. 
If we want to consider the initial boundary value problem of Eq. (1), the initial value is always imposed

$$
u(x, 0)=u_{0}(x), \quad x \in \Omega .
$$

But can we impose Dirichlet homogeneous boundary value

$$
u(x, t)=0,(x, t) \in \partial \Omega \times(0, T),
$$

as usual? When the equation is of weakly degenerate, i.e. there is not interior point in the set $\{s: a(s)=0\}$, we can impose Dirichlet homogeneous boundary condition (4). One can refer to Wu et al. (2001) and the references therein. When the equation is of strongly degenerate, i.e. there is an interior point in the set $\{s: a(s)=0\}$, there are two ways to deal with the corresponding problem. In one way, the entropy solution $u$ is a $B V$ function, which means that

$$
\iint_{Q_{T}}\left|\frac{\partial u}{\partial t}\right| d x d t \leq c, \quad \iint_{Q_{T}}\left|\frac{\partial u}{\partial x_{i}}\right| d x d t \leq c, \quad i=1,2, \ldots, N .
$$

It is well-known that the $B V$ function is the weakest function that one can define the trace on the boundary. In this way, we can directly answer whether (4) is true or not in the sense of the trace, and the general result is that, instead of (4), only a partial boundary value such as

$$
u(x, t)=0,(x, t) \in \Sigma_{1} \times(0, T),
$$

is imposed, where $\Sigma_{1} \subseteq \partial \Omega$ is a relative open subset of $\partial \Omega$. The representative works by Wu and Zhao (1983a, b) had been accomplished in early 1980s, later, one can refer to Yin and Wang (2007). In the other way, the boundary value condition is not directly shown in the sense of the trace as (4), but is elegantly implicitly contained in a family entropy inequalities. Moreover, the entropy solutions defined in this way are only in $L^{\infty}$ space, the existence of the traditional trace [which was called the strong trace in Kobayasi and Ohwa (2012)] on the boundary is not guaranteed, so the boundary value condition is satisfied in a weaker sense than the sense of the trace, one can refer to Carrillo (1999), Li and Qin (2012), Lions et al. (1994), Kobayasi and Ohwa (2012) for more details.

Recently, by the parabolic regularization method, the author Zhan (2015a) had shown the explicit formula of $\Sigma_{1}$ in (5). Let us give some details.

For small $\eta>0$, let

$$
S_{\eta}(s)=\int_{0}^{s} h_{\eta}(\tau) d \tau, h_{\eta}(s)=\frac{2}{\eta}\left(1-\frac{|s|}{\eta}\right)_{+} .
$$

Obviously $h_{\eta}(s) \in C(\mathbb{R})$, and

$$
h_{\eta}(s) \geq 0, \quad\left|s h_{\eta}(s)\right| \leq 1,\left|S_{\eta}(s)\right| \leq 1 ; \lim _{\eta \rightarrow 0} S_{\eta}(s)=\operatorname{sgn} s, \lim _{\eta \rightarrow 0} s S_{\eta}^{\prime}(s)=0 .
$$

Let

$$
\begin{aligned}
& \Sigma_{1 \eta k}=\left\{x \in \Sigma, S_{\eta}(k)\left[b_{i}(0)-b_{i}(k)\right] n_{i}(x)>0\right\}, \\
& \Sigma_{2 \eta k}=\left\{x \in \Sigma, S_{\eta}(k)\left[b_{i}(0)-b_{i}(k)\right] n_{i}(x) \leq 0\right\} .
\end{aligned}
$$


Here and in what follows, $\left\{n_{i}\right\}_{i=1}^{N}$ is the inner normal vector of $\Omega$. Clearly, $\partial \Omega=\Sigma=\Sigma_{1 \eta k} \bigcup \Sigma_{2 \eta k}$. Then

$$
\Sigma_{1}=\bigcup_{\forall \eta \geq 0, \forall k \in \mathbb{R}} \Sigma_{1 \eta k}, \quad \Sigma_{2}=\Sigma \backslash \Sigma_{1}
$$

Basing on (9), if the domain $\Omega$ is bounded, the existence of the entropy solution had been proved in Zhan (2015a). Assuming that

$$
|\triangle d| \leq c, \quad \frac{1}{\lambda} \int_{\Omega_{\lambda}} d x d t \leq c,
$$

the stability of the solutions also had been proved in Zhan (2015a). Here $d(x)=\operatorname{dist}(x, \partial \Omega)$, and $\Omega_{\lambda}=\{x \in \Omega, d(x)<\lambda\}$, when $\lambda$ is small enough. If the domain $\Omega=\mathbb{R}_{+}^{N}$ is the half space of $\mathbb{R}^{N}$, recently in Zhan (2015b), the author had shown that if $b_{N}^{\prime}(0)<0$, then, $\Sigma_{1}=\partial \mathbb{R}_{+}^{N}$, we can impose Dirichlet boundary value

$$
u(x, t)=0,(x, t) \in \partial \mathbb{R}_{+}^{N} \times(0, T) .
$$

But if $b_{N}^{\prime}(0) \geq 0$, then, $\Sigma_{1}=\emptyset$, no any boundary value condition is necessary, the solution of the equation is free from any limitation of the boundary value condition.

Now, inspired by Zhan (2004, 2015a, b) and Zhao and Zhan (2005), we give a new definition of the entropy solution.

Definition 1 A function $u$ is said to be the entropy solution of Eq. (1) with the initial value (3) and with the partial boundary value (5), if

1. $u$ satisfies

$$
u \in B V\left(Q_{T}\right) \cap L^{\infty}\left(Q_{T}\right), \quad \frac{\partial}{\partial x_{i}} \int_{0}^{u} \sqrt{a(s)} d s \in L^{2}\left(Q_{T}\right) .
$$

2. For any $\varphi \in C_{0}^{2}\left(Q_{T}\right), \varphi \geq 0$, for any $k \in \mathbb{R}$, for any small $\eta>0$, u satisfies

$$
\iint_{Q_{T}}\left[I_{\eta}(u-k) \varphi_{t}-B_{\eta}^{i}(u, k) \varphi_{x_{i}}+A_{\eta}(u, k) \Delta \varphi-S_{\eta}^{\prime}(u-k)\left|\nabla \int_{0}^{u} \sqrt{a(s)} d s\right|^{2} \varphi\right] d x d t \geq 0,
$$

where

$$
B_{\eta}^{i}(u, k)=\int_{k}^{u} b_{i}^{\prime}(s) S_{\eta}(s-k) d s, A_{\eta}(u, k)=\int_{k}^{u} a(s) S_{\eta}(s-k) d s, I_{\eta}(u-k)=\int_{0}^{u-k} S_{\eta}(s) d s .
$$

3. The homogeneous boundary value (5) is satisfied in the sense of that

$$
\left.\gamma u\right|_{\Sigma_{1 \eta k}}=0
$$

for any $k, \eta$. Here $\gamma u$ means that the equality is true in the sense of the trace.

4. If the domain $\Omega$ is bounded, the initial value is true in the sense that

$$
\lim _{t \rightarrow 0} \int_{\Omega}\left|u(x, t)-u_{0}(x)\right| d x=0, \text { a.e. } x \in \Omega \text {. }
$$


If the domain is unbounded, the initial value is true in the sense that

$\lim _{t \rightarrow 0} \int_{\Omega_{R}}\left|u(x, t)-u_{0}(x)\right| d x=0$, a.e. $x \in \Omega$,

where $\Omega_{R}=B(0, R) \bigcap \Omega$.

The existence of the entropy solution in the sense of Definition 1 can be proved similar as that in Zhan (2015a), we omit the details here. In our paper, we are mainly concern with the stability of the entropy solutions of Eq. (1) without the condition (10). For simplicity, only some special domains, for examples, the unite $n$-dimensilnal cube

$$
D_{1}=\left\{\left(x_{1}, x_{2}, \ldots, x_{N}\right): 0<x_{i}<1, i=1,2, \ldots, N\right\} .
$$

and the half space $\mathbb{R}_{+}^{N}$, are considered. By choosing special test functions, we will prove the following theorem.

Theorem 1 Suppose that $A(s)$ and $b_{i}(s)$ are smooth enough, $\Omega=D_{1}$ is the unite ndimensilnal cube. If $\Sigma_{1}$ is a subset of $\Sigma$, let $u, v$ be solutions of Eq. (1) with the different initial values $u_{0}(x), v_{0}(x) \in L^{\infty}(\Omega)$ respectively. Suppose that

$$
\gamma u(x, t)=f(x, t), \gamma v=g(x, t),(x, t) \in \Sigma \times(0, T),
$$

and in particular,

$$
\gamma u=\gamma v=0, x \in \Sigma_{1} .
$$

Then

$$
\int_{\Omega}|u(x, t)-v(x, t)| d x \leq \int_{\Omega}\left|u_{0}-v_{0}\right| d x+\text { ess } \sup _{(x, t) \in \Sigma_{2} \times(0, T)}|f(x, t)-g(x, t)|,
$$

where $(x, t) \in \mathbb{R}^{N+1}$, ess $\sup _{(x, t) \in \Sigma_{2} \times(0, T)}|f(x, t)-g(x, t)|$ is in the sense of $N$-dimensonal Hausdorff measure.

Compared Theorem 1 to the results obtained in Zhan (2015a), the essential innovation lies in that, without the condition (10), by skillfully constructing the testing function, we still can obtain the stability. At the last section, we also study the similar problem on half space $\mathbb{R}_{+}^{N}$ and get the similar result, this result is just the same as that in Zhan (2015b), but we supply a simpler proof.

Now, let us give some analysis in the boundary value condition (5) or (12) to see the rationality. By the definition of $\Sigma_{1 \eta k}$, we know that

$$
0<S_{\eta}(k)\left[b_{i}(0)-b_{i}(k)\right] n_{i}=-k S_{\eta}(k) b_{i}^{\prime}(\zeta) n_{i}, \quad x \in \Sigma
$$

where $\zeta \in(k, 0)$. If we let $\eta \rightarrow 0$. Then

$$
b_{i}^{\prime}(\zeta) n_{i}(x)<0, \quad x \in \Sigma .
$$

Let $k \rightarrow 0$. We have

$$
b_{i}^{\prime}(0) n_{i}(x)<0, \quad x \in \Sigma .
$$

The last inequality (17) is in according with the classical Fichrea-Oleinik theory, one can refer to the explanation in previous works (Zhan 2015a, b) 
Let us come back our definition. On the unite $n$-dimensilnal cube $D_{1}$, according to the homogeneous boundary value condition (5), and by (17), we have

$$
\gamma u=\gamma v=0, x \in \bigcup_{i=1}^{N}\left\{x \in \partial D_{1}:\left(x_{i}-\frac{1}{2}\right) b_{i}^{\prime}(0)>0\right\} .
$$

For example, when we consider the boundary plane $\left\{x_{N}=0\right\}, \mathbf{n}=\{0,0, \ldots, 0,1\}$, (17) implies $b_{i}^{\prime}(0) n_{i}=b_{N}^{\prime}(0)<0,(18)$ is true on $\left\{x_{N}=0\right\}$, we should give the boundary value on $\left\{x_{N}=0\right\}$, while, on $\left\{x_{N}=1\right\}$ we can not give the boundary value. If $b_{N}^{\prime}(0)>0$, then (18) is true on $\left\{x_{N}=1\right\}$, which implies that we should give the boundary value on $\left\{x_{N}=1\right\}$, while, on $\left\{x_{N}=0\right\}$ we can not give the boundary value. Certainly, if $b_{N}^{\prime}(0)=0$, both on $\left\{x_{N}=0\right\}$ and on $\left\{x_{N}=1\right\}$, no boundary value condition is imposed.

\section{Kruzkov's bi-variables method}

Let $\Gamma_{u}$ be the set of all jump points of $u \in B V\left(Q_{T}\right), v$ the normal of $\Gamma_{u}$ at $X=(x, t), u^{+}(X)$ and $u^{-}(X)$ the approximate limits of $u$ at $X \in \Gamma_{u}$ with respect to $(v, Y-X)>0$ and $(v, Y-X)<0$ respectively. For continuous function $p(u, x, t)$ and $u \in B V\left(Q_{T}\right)$, define

$$
\widehat{p}(u, x, t)=\int_{0}^{1} p\left(\tau u^{+}+(1-\tau) u^{-}, x, t\right) d \tau,
$$

which is called the composite mean value of $p$. For a given $t$, we denote $\Gamma_{u}^{t}, H^{t},\left(v_{1}^{t}, \ldots, v_{N}^{t}\right)$ and $u_{ \pm}^{t}$ as all jump points of $u(\cdot, t)$, Housdorff measure of $\Gamma_{w}^{t}$, the unit normal vector of $\Gamma_{u}^{t}$, and the asymptotic limit of $u(\cdot, t)$ respectively. Moreover, if $f(s) \in C^{1}(\mathbb{R}), u \in B V\left(Q_{T}\right)$, then $f(u) \in B V\left(Q_{T}\right)$ and

$$
\frac{\partial f(u)}{\partial x_{i}}=\widehat{f}^{\prime}(u) \frac{\partial u}{\partial x_{i}}, \quad i=1,2, \ldots, N, N+1,
$$

where $x_{N+1}=t$ as usual.

Lemma 1 Let $u$ be the solution of Eq. (1) in the sense of Definition 1. Then

$$
a(s)=0, s \in I\left(u^{+}(x, t), u^{-}(x, t)\right) \text { a.e. on } \Gamma_{u},
$$

which $I(\alpha, \beta)$ denote the closed interval with endpoints $\alpha$ and $\beta$, and (21) is in the sense of Hausdorff measure $H_{N}\left(\Gamma_{u}\right)$.

This lemma can be proved in a similar way as Zhan (2004); Zhao and Zhan (2005), we omit the details here.

Now, we will show that how Kruzkov's bi-variables method, which was used to deal with the conservation law equation (Kružkov 1970) originally, can be used to prove the stability of the solutions to Eq. (1). Let $u, v$ be two entropy solutions of Eq. (1) with initial values

$$
u(x, 0)=u_{0}(x), v(x, 0)=v_{0}(x),
$$

and with the boundary values (14)-(15), in particular, $u(x, t)=v(x, t)=0$, $(x, t) \in \Sigma_{1} \times(0, T)$. 
By Definition 1 , for $\varphi \in C_{0}^{2}\left(Q_{T}\right)$, we have

$$
\begin{gathered}
\iint_{Q_{T}}\left[I_{\eta}(u-k) \varphi_{t}-B_{\eta}^{i}(u, k) \varphi_{x_{i}}+A_{\eta}(u, k) \Delta \varphi-S_{\eta}^{\prime}(u-k)\left|\nabla \int_{0}^{u} \sqrt{a(s)} d s\right|^{2} \varphi\right] d x d t \geq 0, \\
\iint_{Q_{T}}\left[I_{\eta}(v-l) \varphi_{\tau}-B_{\eta}^{i}(v, l) \varphi_{y_{i}}+A_{\eta}(v, l) \Delta \varphi-S_{\eta}^{\prime}(v-l)\left|\nabla \int_{0}^{v} \sqrt{a(s)} d s\right|^{2} \varphi\right] d y d \tau \geq 0 .
\end{gathered}
$$

Let $\psi(x, t, y, \tau)=\phi(x, t) j_{h}(x-y, t-\tau)$, where $\phi(x, t) \geq 0, \phi(x, t) \in C_{0}^{\infty}\left(Q_{T}\right)$, and

$$
j_{h}(x-y, t-\tau)=\omega_{h}(t-\tau) \Pi_{i=1}^{N} \omega_{h}\left(x_{i}-y_{i}\right) .
$$

Here

$$
\omega_{h}(s)=\frac{1}{h} \omega\left(\frac{s}{h}\right), \omega(s) \in C_{0}^{\infty}(R), \omega(s) \geq 0, \omega(s)=0 \text { if }|s|>1, \int_{-\infty}^{\infty} \omega(s) d s=1 .
$$

We choose $k=v(y, \tau), l=u(x, t), \varphi=\psi(x, t, y, \tau)$ in (22) (23), integrate over $Q_{T}$, then

$$
\begin{aligned}
& \iint_{Q_{T}} \iint_{Q_{T}}\left[I_{\eta}(u-v)\left(\psi_{t}+\psi_{\tau}\right)-\left(B_{\eta}^{i}(u, v) \psi_{x_{i}}+B_{\eta}^{i}(v, u) \psi_{y_{i}}\right)+A_{\eta}(u, v) \Delta_{x} \psi+A_{\eta}(v, u) \Delta_{y} \psi\right] \\
& -S_{\eta}^{\prime}(u-v)\left(\left|\nabla \int_{0}^{u} \sqrt{a(s)} d s\right|^{2}+\left|\nabla \int_{0}^{v} \sqrt{a(s)} d s\right|^{2}\right) \psi d x d t d y d \tau=0 .
\end{aligned}
$$

Clearly,

$$
\frac{\partial j_{h}}{\partial t}+\frac{\partial j_{h}}{\partial \tau}=0, \quad \frac{\partial j_{h}}{\partial x_{i}}+\frac{\partial j_{h}}{\partial y_{i}}=0, \quad i=1, \ldots, N ; \quad \frac{\partial \psi}{\partial t}+\frac{\partial \psi}{\partial \tau}=\frac{\partial \phi}{\partial t} j_{h}, \quad \frac{\partial \psi}{\partial x_{i}}+\frac{\partial \psi}{\partial y_{i}}=\frac{\partial \phi}{\partial x_{i}} j_{h} .
$$

Noticing that

$$
\lim _{\eta \rightarrow 0} B_{\eta}^{i}(u, v)=\lim _{\eta \rightarrow 0} B_{\eta}^{i}(v, u)=\operatorname{sgn}(u-v)\left(b_{i}(u)-b_{i}(v)\right),
$$

as $\eta \rightarrow 0$, we have,

$$
\begin{aligned}
& \iint_{Q_{T}} \iint_{Q_{T}}\left[B_{\eta}^{i}(u, v) \psi_{x_{i}}+B_{\eta}^{i}(v, u) \psi_{y_{i}}\right] d x d t d y d \tau \\
& \quad \rightarrow \iint_{Q_{T}} \iint_{Q_{T}} \operatorname{sgn}(u-v)\left[b_{i}(u)-b_{i}(v)\right] \phi_{x_{i}} j_{h} d x d t d y d \tau,
\end{aligned}
$$

as $h \rightarrow 0$, we have

$$
\begin{gathered}
\iint_{Q_{T}} \iint_{Q_{T}} \operatorname{sgn}(u-v)\left[b_{i}(u)-b_{i}(v)\right] \phi_{x_{i}} j_{h} d x d t d y d \tau \\
\rightarrow \iint_{Q_{T}} \operatorname{sgn}(u-v)\left[b_{i}(u)-b_{i}(v)\right] \phi_{x_{i}} d x d t .
\end{gathered}
$$

At the same time, we have 


$$
\begin{aligned}
& \iint_{Q_{T}} \iint_{Q_{T}} S_{\eta}^{\prime}(u-v)\left(\left|\nabla_{x} \int_{0}^{u} \sqrt{a(s)} d s\right|^{2}+\left|\nabla_{y} \int_{0}^{v} \sqrt{a(s)} d s\right|^{2}\right) \psi d x d t d y d \tau \\
& =\iint_{Q_{T}} \iint_{Q_{T}} S_{\eta}^{\prime}(u-v)\left(\left|\nabla_{x} \int_{0}^{u} \sqrt{a(s)} d s\right|-\left|\nabla_{y} \int_{0}^{v} \sqrt{a(s)} d s\right|\right)^{2} \psi d x d t d y d \tau \\
& \quad+2 \iint_{Q_{T}} \iint_{Q_{T}} S_{\eta}^{\prime}(u-v) \nabla_{x} \int_{0}^{u} \sqrt{a(s)} d s \cdot \nabla_{y} \int_{0}^{v} \sqrt{a(s)} d s \psi d x d t d y d \tau,
\end{aligned}
$$

and

$$
\begin{aligned}
& \iint_{Q_{T}}\left[A_{\eta}(u, v) \Delta_{x} \psi+A_{\eta}(v, u) \Delta_{y} \psi\right] d x d t d y d \tau \\
& =\iint_{Q_{T}} \iint_{Q_{T}}\left\{A_{\eta}(u, v)\left(\Delta_{x} \phi j_{h}+2 \phi_{x_{i}} j_{h x_{i}}+\phi \Delta j_{h}\right)+A_{\eta}(v, u) \phi \Delta_{y} j_{h}\right\} d x d t d y d \tau \\
& =\iint_{Q_{T}} \iint_{Q_{T}}\left\{A_{\eta}(u, v) \Delta_{x} \phi j_{h}+A_{\eta}(u, v) \phi_{x_{i}} j_{h x_{i}}+A_{\eta}(v, u) \phi_{x_{i}} j_{h y_{i}}\right\} d x d t d y d \tau \\
& \quad-\iint_{Q_{T}} \iint_{Q_{T}}\left\{a(u) \widehat{S_{\eta}(u}-v\right) \frac{\partial u}{\partial x_{i}}-\int_{u}^{v} \widehat{\left.\left.a(s) S_{\eta}^{\prime}(s-v) d s \frac{\partial u}{\partial x_{i}}\right) \phi j_{h x_{i}}\right\} d x d t d y d \tau,}
\end{aligned}
$$

where

$$
\begin{aligned}
\left.a(u) \widehat{S_{\eta}(u}-v\right) & =\int_{0}^{1} a\left(s u^{+}+(1-s) u^{-}\right) S_{\eta}\left(s u^{+}+(1-s) u^{-}-v\right) d s, \int_{u}^{v} a(s) \widehat{S_{\eta}^{\prime}(s-v) d s} \\
& =\int_{0}^{1} \int_{s u^{+}+(1-s) u^{-}}^{v} a(\sigma) S_{\eta}\left(\sigma-s u^{+}-(1-s) u^{-}\right) d \sigma d s .
\end{aligned}
$$

Now, we will combine the last term on the right hand of (28) with the last term on the right hand side of (29). In details, by Lemma 1, at one hand, we have

$$
\begin{aligned}
\iint_{Q_{T}} \iint_{Q_{T}} \nabla_{x} \nabla_{y} \int_{v}^{u} \sqrt{a(\delta)} \int_{\delta}^{v} \sqrt{a(\sigma)} S_{\eta}^{\prime}(\sigma-\delta) d \sigma d \delta \psi d x d t d y d \tau \\
=\iint_{Q_{T}} \iint_{Q_{T}} \int_{0}^{1} \int_{0}^{1} \sqrt{a\left(s u^{+}+(1-s) u^{-}\right)} \sqrt{a\left(\sigma v^{+}+(1-\sigma) v^{-}\right)} \\
\quad \times S_{\eta}^{\prime}\left[\sigma v^{+}+(1-\sigma) v^{-}-s u^{+}-(1-s) u^{-}\right] d s d \sigma \nabla_{x} u \nabla_{y} v d x d t d y d \tau \\
=\iint_{Q_{T}} \iint_{Q_{T}} \int_{0}^{1} \int_{0}^{1} S_{\eta}^{\prime}\left[\sigma v^{+}+(1-\sigma) v^{-}-s u^{+}-(1-s) u^{-}\right] d s d \sigma \\
\quad \times \sqrt{a(u)} \nabla_{x} u \sqrt{a(v)} \nabla_{y} v d x d t d y d \tau \\
=\iint_{Q_{T}} \iint_{Q_{T}} \int_{0}^{1} \int_{0}^{1} S_{\eta}^{\prime}(v-u) \nabla_{x} \int_{0}^{u} \sqrt{a(s)} d s \nabla_{y} \int_{0}^{v} \sqrt{a(s)} d s d x d t d y d \tau .
\end{aligned}
$$

At the other hand, we have

$$
\begin{aligned}
& \iint_{Q_{T}} \iint_{Q_{T}} \nabla_{x} \nabla_{y} \int_{v}^{u} \sqrt{a(\delta)} \int_{\delta}^{v} \sqrt{a(\sigma)} S_{\eta}^{\prime}(\sigma-\delta) d \sigma d \delta \psi d x d t d y d \tau \\
& =\iint_{Q_{T}} \iint_{Q_{T}} \int_{0}^{1} \sqrt{a\left(s u^{+}+(1-s) u^{-}\right)} \\
& \quad \times \int_{s u^{+}+(1-s) u^{-}}^{v} \sqrt{a(\sigma)} S_{\eta}^{\prime}\left(\sigma-s u^{+}-(1-s) u^{-}\right) d \sigma d s \frac{\partial u}{\partial x_{i}} j_{h x_{i}} \phi d x d t d y d \tau .
\end{aligned}
$$


By (30) (31), we have

$$
\begin{aligned}
\iint_{Q_{T}} & \left.\iint_{Q_{T}}\left(a(u) \widehat{S_{\eta}(u}-v\right) \frac{\partial u}{\partial x_{i}}-\int_{u}^{v} a(s) S_{\eta}^{\prime}(s-u) d s \frac{\partial u}{\partial x_{i}}\right) j_{h x_{i}} \phi d x d t d y d \tau \\
& +2 \iint_{Q_{T}} \iint_{Q_{T}} S_{\eta}^{\prime}(u-v) \nabla_{x} \int_{0}^{u} \sqrt{a(s)} d s \cdot \nabla_{y} \int_{0}^{v} \sqrt{a(s)} d s \psi d x d t d y d \tau \\
= & \iint_{Q_{T}} \iint_{Q_{T}}\left[\int_{0}^{1} a\left(s u^{+}+(1-s) u^{-}\right) S_{\eta}\left(s u^{+}+(1-s) u^{-}-v\right) d s\right. \\
& -\int_{0}^{1} \int_{s u^{+}+(1-s) u^{-}}^{v} a(\sigma) S_{\eta}^{\prime}\left(\sigma-s u^{+}-(1-s) u^{-}\right) d \sigma d s \\
& +2 \int_{0}^{1} \sqrt{a\left(s u^{+}+(1-s) u^{-}\right)} \int_{s u^{+}+(1-s) u^{-}}^{v} \sqrt{a(\sigma)} \\
& \left.\times S_{\eta}^{\prime}\left(\sigma-s u^{+}-(1-s) u^{-}\right) d \sigma d s\right] \frac{\partial u}{\partial x_{i}} j_{h x_{i}} \phi d x d t d y d \tau \\
= & -\iint_{Q_{T}} \iint_{Q_{T}} \int_{0}^{1} \int_{s u^{+}+(1-s) u^{-}}^{v}\left[\sqrt{a(\sigma)}-\sqrt{a\left(s u^{+}+(1-s) u^{-}\right)}\right] \\
& \times S_{\eta}^{\prime}\left(\sigma-s u^{+}-(1-s) u^{-}\right) d \sigma d s \frac{\partial u}{\partial x_{i}} j_{h x_{i}} \phi d x d t d y d \tau \rightarrow 0,
\end{aligned}
$$

as $\eta \rightarrow 0$.

Let us come back (29). Since

$$
\lim _{\eta \rightarrow 0} A_{\eta}(u, v)=\lim _{\eta \rightarrow 0} A_{\eta}(v, u)=\operatorname{sgn}(u-v)[A(u)-A(v)]
$$

we have

$$
\lim _{\eta \rightarrow 0}\left[A_{\eta}(u, v) \phi_{x_{i}} j_{h x_{i}}+A_{\eta}(u, v) \phi_{y_{i}} j_{h y_{i}}\right]=0
$$

Combing (26)-(28) with (32)-(33), and letting $\eta \rightarrow 0, h \rightarrow 0$ in (26). We obtain

$$
\begin{aligned}
& \iint_{Q_{T}}\left[|u(x, t)-v(x, t)| \phi_{t}-\operatorname{sgn}(u-v)\left(b_{i}(u)-b_{i}(v)\right) \phi_{x_{i}}+|A(u)-A(v)| \Delta \phi\right] d x d t \\
& \quad=\iint_{Q_{T}} \iint_{Q_{T}} S_{\eta}^{\prime}(u-v)\left(\left|\nabla_{x} \int_{0}^{u} \sqrt{a(s)} d s\right|-\left|\nabla_{y} \int_{0}^{v} \sqrt{a(s)} d s\right|\right)^{2} \psi d x d t d y d \tau \geq 0 .
\end{aligned}
$$

By Kruzkov's bi-variables method it means that, by a process of limit, we can choose a suitable test function $\phi \in C_{0}^{1}\left(Q_{T}\right)$ in (34), to obtain the stability of the solutions.

\section{Proof of Theorem 1}

The proof of Theorem 1 Let $x=\left\{x_{1}, x_{2}, \ldots, x_{i}, \ldots, N\right\}$ and define

$$
d_{i}\left(x_{i}\right)= \begin{cases}x_{i}, & \text { if } 0 \leq x_{i} \leq \frac{1}{2} \\ 1-x_{i}, & \text { if } \frac{1}{2}<x_{i} \leq 1\end{cases}
$$

For small enough $\lambda$, we set

$$
\varphi_{i \lambda}\left(x_{i}\right)=\left\{\begin{array}{cc}
\sin \frac{1}{\lambda}\left(d_{i}\left(x_{i}\right)\right), & \text { if } 0 \leq d_{i}\left(x_{i}\right) \leq \frac{\pi \lambda}{2} \\
1, & \text { if } d_{i}(x) \geq \frac{\pi \lambda}{2}
\end{array}\right.
$$


Let $0 \leq \eta(t) \in C_{0}^{1}(t)$ and choose the test function in (34) as

$$
\phi(x, t)=\eta(t) \prod_{j=1}^{N} \varphi_{j \lambda}\left(x_{j}\right)
$$

Then

$$
\begin{aligned}
\partial_{x_{i}} \phi(x, t) & =\eta(t) \partial_{x_{i}} \varphi_{i \lambda}\left(x_{i}\right) \prod_{j=1, j \neq i}^{N} \varphi_{j \lambda}\left(x_{j}\right) \\
& =\eta(t) \frac{1}{\lambda} \cos \frac{1}{\lambda}\left(d_{i}\left(x_{i}\right)\right) d_{i x_{i}}\left(x_{i}\right) \prod_{j=1, j \neq i}^{N} \varphi_{j \lambda}\left(x_{j}\right), \quad 0 \leq d_{i}\left(x_{i}\right) \leq \frac{\pi \lambda}{2} . \\
\triangle \phi(x, t) & =\frac{1}{\lambda} \eta(t) \prod_{j=1, j \neq i}^{N} \varphi_{j \lambda}\left(x_{j}\right)\left[-\frac{1}{\lambda} \sin \frac{1}{\lambda}\left(d_{i}\left(x_{i}\right)\right) d_{i x_{i}}^{2}+\frac{1}{\lambda} \cos \frac{1}{\lambda}\left(d_{i}\left(x_{i}\right)\right) \triangle d_{i}\left(x_{i}\right)\right] \\
& =-\frac{1}{\lambda^{2}} \eta(t) \prod_{j=1, j \neq i}^{N} \varphi_{j \lambda}\left(x_{j}\right) \sin \frac{1}{\lambda}\left(d_{i}\left(x_{i}\right)\right) d_{i x_{i}}^{2} \leq 0, \quad 0 \leq d_{i}\left(x_{i}\right) \leq \frac{\pi \lambda}{2} .
\end{aligned}
$$

For (39) in (34),

$$
\iint_{Q_{T}}\left[|u(x, t)-v(x, t)| \phi_{t}-\operatorname{sgn}(u-v)\left(b_{i}(u)-b_{i}(v)\right) \partial_{x_{i}} \phi(x, t)\right] d x d t \geq 0 .
$$

By that $\left|\partial_{x_{i}} \phi(x, t)\right| \leq \frac{1}{\lambda},\left|b_{i}(u)-b_{i}(v)\right| \leq c|u-v|$, we have

$$
\iint_{Q_{T}}|u(x, t)-v(x, t)| \phi_{t} d x d t+c \int_{0}^{T} \int_{\Omega_{\lambda}} \eta(t) \frac{1}{\lambda} \| u-v \mid d x d t \geq 0,
$$

where $\Omega_{\lambda}=\left\{x \in \Omega: d_{i}\left(x_{i}\right)<\frac{\lambda \pi}{2}\right\}$. According to the definition of the trace of BV functions (Enrico 1984), when $x \in \Sigma_{1}, \gamma u=\gamma v=0$, let $\lambda \rightarrow 0$ in (41). We have

$$
\begin{aligned}
& \lim _{\lambda \rightarrow 0} \int_{0}^{T} \eta(t) \frac{1}{\lambda} \int_{\Omega_{\lambda}}|| u-v\left|d x d t=c \int_{0}^{T} \eta(t)\right| u-\left.v\right|_{\partial \Omega} d t \\
& \quad=c \int_{0}^{T} \eta(t)|u-v|_{\partial \Omega} d t \leq c \cdot \operatorname{essup}_{\Sigma_{2} \times(0, T)}|f(x, t)-g(x, t)| .
\end{aligned}
$$

Let $\lambda \rightarrow 0$ in (41). Then

$$
c \cdot \operatorname{essup}_{\Sigma_{2} \times(0, T)}|f(x, t)-g(x, t)|+\iint_{Q_{T}}|u(x, t)-v(x, t)| \eta_{t}^{\prime} d x d t \geq 0 .
$$

Let $0<s<\tau<T$, and

$$
\eta(t)=\int_{\tau-t}^{s-t} \alpha_{\varepsilon}(\sigma) d \sigma, \varepsilon<\min \{\tau, T-s\}
$$

Here $\alpha_{\varepsilon}(t)$ is the kernel of mollifier with $\alpha_{\varepsilon}(t)=0$ for $t \notin(-\varepsilon, \varepsilon)$. Then

$$
c \cdot \operatorname{essup}_{\Sigma_{2} \times(0, T)}|f(x, t)-g(x, t)|+\int_{0}^{T}\left[\alpha_{\varepsilon}(t-s)-\alpha_{\varepsilon}(t-\tau)\right]|u-v|_{L^{1}(\Omega)} d t \geq 0
$$


Let $\varepsilon \rightarrow 0$. Then

$$
|u(x, \tau)-v(x, \tau)|_{L^{1}(\Omega)} \leq|u(x, s)-v(x, s)|_{L^{1}(\Omega)}+c \cdot \operatorname{essup}_{\Sigma_{2} \times(0, T)}|f(x, t)-g(x, t)|
$$

and the desired result follows by letting $s \rightarrow 0$.

\section{On the half space}

At the last section of the paper, let's consider Eq. (1) on the half space

$$
\frac{\partial u}{\partial t}=\Delta A(u)+\operatorname{div}(b(u)),(x, t) \in \mathbb{R}_{+}^{N} \times(0, T),
$$

with the initial value condition

$$
u(x, 0)=u_{0}(x), x \in \mathbb{R}_{+}^{N},
$$

where $\mathbb{R}_{+}^{N}=\left\{x=\left(x_{1}, x_{2}, \ldots, x_{N}\right): x_{N}>0\right\}$ is the half space of $\mathbb{R}^{N}$. The author Zhan (2015b) had shown that when $b_{N}^{\prime}(0)<0$, we can give Direchlet homogeneous boundary value

$$
u(x, t)=0,(x, t) \in \partial \mathbb{R}_{+}^{N} \times(0, T)=\Sigma \times(0, T) .
$$

while $b_{N}^{\prime}(0) \geq 0$, no any boundary value condition is necessary. Now, we give the definitions of the entropy solutions of the Eq. (43), which are the minor versions of the Definition 2.1 in Zhan (2015b).

Definition 2 Let $b_{N}^{\prime}(0)<0$. A function $u$ is said to be the entropy solution of Eq. (43) with the initial value (44) and the boundary value (45), if

1. $u$ satisfies

$$
u \in B V\left(Q_{T}\right) \cap L^{\infty}\left(Q_{T}\right) \cap L^{1}\left(Q_{T}\right), \frac{\partial}{\partial x_{i}} \int_{0}^{u} \sqrt{a(s)} d s \in L^{2}\left(Q_{T}\right) .
$$

2. For any $\varphi, \varphi \in C_{0}^{2}\left(Q_{T}\right), \varphi \geq 0$, for any $k \in \mathbb{R}$, for any small $\eta>0$, $u$ satisfies

$$
\begin{gathered}
\iint_{Q_{T}}\left[I_{\eta}(u-k) \varphi_{t}-B_{\eta}^{i}(u, k) \varphi_{x_{i}}+A_{\eta}(u, k) \Delta \varphi\right. \\
\left.-S_{\eta}^{\prime}(u-k)\left|\nabla \int_{0}^{u} \sqrt{a(s)} d s\right|^{2} \varphi\right] d x d t \geq 0 .
\end{gathered}
$$

3. For any positive constant $R$,

$$
\lim _{t \rightarrow 0} \int_{B_{R}\left(x^{R}\right)}\left|u(x, t)-u_{0}(x)\right| d x=0 .
$$

for any given positive constant $R$, where $x^{R}=(0,0, \ldots, 0, R), \quad B_{R}\left(x^{R}\right)=$ $\left\{x \in \mathbb{R}_{+}^{N}:\left|x-x^{R}\right|<R\right\}$.

4. The boundary value condition (45) is true in the sense that the traces $\gamma u=\gamma \nu=0$ on $\partial \mathbb{R}_{+}^{N}$ as usual.

Definition 3 Let $b_{N}^{\prime}(0) \geq 0$. A function $u$ is said to be the entropy solution of Eq. (43) with the initial value (44), if $u$ satisfies Definition 2 except the fourth point. In this case, no boundary value condition is required. 
Now, we actually are able to prove the existence of the solutions defined as Definition 2-3 in a similar way as Zhan (2015b), we omit the details here. In what follows, we only provide a new and simpler proof of the stability of the solutions.

Theorem 2 Suppose that $A(s)$ and $b_{i}(s)$ are smooth enough. Let $u, v$ be solutions of Eq. (1) with the different initial values $u_{0}(x), v_{0}(x) \in L^{\infty}\left(\mathbb{R}_{+}^{N}\right) \cap L^{1}\left(\mathbb{R}_{+}^{N}\right)$ respectively. If $b_{N}^{\prime}(0)<0$, suppose that the traces $\gamma u=\gamma v=0$ on $\Sigma$ as (45). Then

$$
\int_{\mathbb{R}_{+}^{N}}|u(x, t)-v(x, t)| d x \leq \int_{\mathbb{R}_{+}^{N}}\left|u_{0}-v_{0}\right| d x
$$

If $b_{N}^{\prime}(0) \geq 0$, then

$$
\int_{\mathbb{R}_{+}^{N}}|u(x, t)-v(x, t)| d x \leq \int_{\mathbb{R}_{+}^{N}}\left|u_{0}-v_{0}\right| d x+c \operatorname{esssup}_{\Sigma \times(0, T)}|u(x, t)-v(x, t)| .
$$

Proof By Kruzkov's bi-variables method, as we have done in section “Kruzkov's bi-variables method", we can get

$\iint_{Q_{T}}\left[|u(x, t)-v(x, t)| \phi_{t}-\operatorname{sgn}(u-v)\left(b_{i}(u)-b_{i}(v)\right) \phi_{x_{i}}+|A(u)-A(v)| \Delta \phi\right] d x d t \geq 0$.

Now, we can choose $\phi$ in (50) by

$$
\phi(x, t)=\omega_{\lambda}(x) \eta(t)
$$

where $\eta(t) \in C_{0}^{\infty}(0, T)$, and $\omega_{\lambda}(x) \in C_{0}^{2}(\Omega)$ is defined as follows: for any given small enough $0<\lambda, 0 \leq \omega_{\lambda} \leq 1,\left.\omega\right|_{\partial \Omega}=0$, and when $x_{N} \geq \lambda$,

$$
\omega_{\lambda}(x)=1,
$$

when $0 \leq x_{N} \leq \lambda$,

$$
\omega_{\lambda}(x)=\omega_{\lambda}\left(x_{N}\right)=1-\frac{\left(x_{N}-\lambda\right)^{2}}{\lambda^{2}} .
$$

Clearly,

$\phi_{x_{i}}=\eta(t)\left(\omega_{\lambda}\left(x_{N}\right)\right)_{x_{i}} \leq c\left|\omega_{\lambda}^{\prime}\left(x_{N}\right)\right| \leq \frac{c}{\lambda} . \Delta \phi=\eta(t) \triangle\left(\omega_{\lambda}\left(x_{N}\right)\right)=\eta(t) \nabla\left(\omega_{\lambda}^{\prime}\left(x_{N}\right) \nabla x_{N}\right)=-\eta(t) \frac{2}{\lambda^{2}}$.

Then by (50),

$$
\begin{aligned}
0 \leq & \iint_{Q_{T}}|u(x, t)-v(x, t)| \eta^{\prime}(t) \omega_{\lambda}(x) d x d t \\
& -\iint_{Q_{T}}\left[\operatorname{sgn}(u-v)\left(b_{N}(u)-b_{N}(v)\right) \eta(t) \frac{\partial \omega_{\lambda}(x)}{\partial x_{N}}+|A(u)-A(v)| \eta(t) \Delta \omega_{\lambda}(x)\right] d x d t \\
\leq & \iint_{Q_{T}}|u(x, t)-v(x, t)| \eta^{\prime}(t) \omega_{\lambda}(x) d x d t+c \int_{0}^{T} \eta(t) d t \frac{1}{\lambda} \int_{\left\{x_{N}<\lambda\right\}}|u-v| d x .
\end{aligned}
$$


Noticing $\lim _{\lambda \rightarrow 0} \omega_{\lambda}=1$, by the definition of the trace of a BV function, let $\lambda \rightarrow 0$ in (51).

Then

$$
\iint_{Q_{T}}|u(x, t)-v(x, t)| \eta^{\prime}(t) d x d t+c \operatorname{essup}|u-v|_{\Sigma \times(0, T)} \geq 0 .
$$

Let $0<s<\tau<T$, and

$$
\eta(t)=\int_{\tau-t}^{s-t} \alpha_{\epsilon}(\sigma) d \sigma, \epsilon<\min \{\tau, T-s\}
$$

as before. Let $\epsilon \rightarrow 0$. Then

$$
\int_{\Omega}|u(x, s)-v(x, s)| d x \leq \int_{\Omega}|u(x, \tau)-v(x, \tau)| d x+c \operatorname{essup}|| u-\left.v\right|_{\Sigma \times(0, T)} .
$$

Let $s \rightarrow 0$. If $b_{N}^{\prime}(0)<0$, suppose that the traces $\gamma u=\gamma \nu=0$ on $\Sigma$,

$$
\int_{\mathbb{R}_{+}^{N}}|u(x, t)-v(x, t)| d x \leq \int_{\mathbb{R}_{+}^{N}}\left|u_{0}-v_{0}\right| d x .
$$

If $b_{N}^{\prime}(0) \geq 0$, no any boundary value condition is necessary, then

$$
\int_{\mathbb{R}_{+}^{N}}|u(x, t)-v(x, t)| d x \leq \int_{\mathbb{R}_{+}^{N}}\left|u_{0}-v_{0}\right| d x+c \operatorname{esssup}_{\Sigma \times(0, T)}|u(x, t)-v(x, t)| .
$$

we have the conclusion.

\section{Conclusion}

The paper shows that there is an essential difference of the boundary conditions between the strongly degenerate parabolic equation and the weakly degenerate parabolic equation. Instead of the whole boundary $\partial \Omega$, only a part of $\partial \Omega$ on which we can impose the boundary value if the well-posedness of the solutions to a strongly parabolic equation is considered. In physics, for example, if we consider a special case of Eq. (1), we consider the nonlinear heat conduction equation

$$
u_{t}=\operatorname{div}(k(u) \nabla u)
$$

if $k(0)=0$, it means there is not heat flux across the boundary. Then the partial boundary $\Sigma_{1}=\emptyset$, so there is no any boundary condition is necessary.

Acknowledgments

The paper is supported by NSF of China (No. 11371297), supported by NSF of Fujian Province (No: 2015J01592), China.

\section{Competing interests}

The author declares that he has no competing interests.

Received: 9 October 2015 Accepted: 23 September 2016

Published online: 18 October 2016

\section{References}

Bendahmane M, Karlsen KH (2004) Reharmonized entropy solutions for quasilinear anisotropic degenerate parabolic equations. SIAM J Math Anal 36(2):405-422 
Brezis H, Crandall MG (1979) Uniqueness of solutions of the initial value problem for $u_{t}-\Delta \varphi(u)=0$. J Math Pures et Appl 58:153-163

Carrillo J (1999) Entropy solutions for nonlinear degenerate problems. Arch Ration Mech Anal 147:269-361

Chen GQ, Dibenedetto E (2001) Stability of entropy solutions to Cauchy problem for a class of nonlinear hyperbolicparabolic equations. SIAM J Math Anal 33(4):751-762

Chen GQ, Perthame B (2003) Well-posedness for non-isotropic degenerate parabolic-hyperbolic equations. Ann I H Poincare-AN 20:645-668

Cockburn B, Gripenberg G (1999) Continuous dependence on the nonlinearities of solutions of degenerate parabolic equations. J Differ Equ 151:231-251

Enrico G (1984) Minimal surfaces and functions of bounded variation. Birkhauser, Bosten

Evans LC (1998) Weak convergence methods for nonlinear partial differential equations. In: Conference board of the mathematical sciences, regional conferences series in mathematics no. 74

Karlsen KH, Risebro NH (2003) On the uniqueness of entropy solutions of nonlinear degenerate parabolic equations with rough coefficient. Discrete Contain Dye Syst 9(5):1081-1104

Kobayasi K, Ohwa H (2012) Uniqueness and existence for anisotropic degenerate parabolic equations with boundary conditions on a bounded rectangle. J Differ Equ 252:137-167

Kružkov SN (1970) First order quasilinear equations in several independent variables. Math USSR-Sb 10:217-243

Lions PL, Perthame B, Tadmor E (1994) A kinetic formation of multidimensional conservation laws and related equations. J Am Math Soc 7:169-191

Li Y, Wang Q (2012) Homogeneous Dirichlet problems for quasilinear anisotropic degenerate parabolic- hyperbolic equations. J Differ Equ 252:4719-4741

Oleinik OA, Samokhin VN (1999) Mathematical models in boundary layer theorem. Chapman and Hall/CRC, London

Vol'pert Al, Hudjave SI (1975) Analysis of classof discontinuous functions and the equations of mathematical physics(Russian), Izda. Nauka Moskwa

Vol'pert Al (1967) BV space and quasilinear equations. MatSb 73:255-302

Vol'pert Al, Hudjaev SI (1967) On the problem for quasilinear degenerate parabolic equations of second order (Russian). Mat Sb 3:374-396

Wu Z, Zhao J, Yin J, Li H (2001) Nonlinear diffusion equations. Word Scientific Publishing, Singapore

Wu Z, Yin J (1989) Some properties of functions in BV $x$ and their applications to the uniqueness of solutions for degenerate quasilinear parabolic equations. Northeast Math J 5(4):395-422

Wu Z, Zhao J (1983) The first boundary value problem for quasilinear degenerate parabolic equations of second order in several variables. Chin Ann Math 4B(1):57-76

Wu Z, Zhao J (1983) Some general results on the first boundary value problem for quasilinesr degenerate parabolic equations. Chin Ann Math 4B(3):319-328

Yin J, Wang C (2007) Evolutionary weighted p-Laplacian with boundary degeneracy. J Differ Equ 237:421-445

Zhan $\mathrm{H}$ (2004) The study of the Cauchy problem of a second order quasilinear degenerate parabolic equation and the parallelism of a Riemannian manifold, Doctor Thesis, Xiamen University

Zhan H (2015) Homogeneous Dirichlet condition of aanisotropic degenerate parabolic equation. Bound Value Probl 22(2015):1-22

Zhan H (2015) The solutions of a hyperbolic-parabolic mixed type equation on half-space domain. J Differ Equ 259:1449-1481

Zhao J (1985) Uniqueness of solutions of quasilinear degenerate parabolic equations. Northeastern Math J 1(2):153-165

Zhao J, Zhan H (2005) Uniqueness and stability of solution for Cauchy problem of degenerate quasilinear parabolic equations, Sci China Ser A 48:583-593

\section{Submit your manuscript to a SpringerOpen ${ }^{\circ}$ journal and benefit from:}

- Convenient online submission

Rigorous peer review

- Immediate publication on acceptance

Open access: articles freely available online

- High visibility within the field

Retaining the copyright to your article

Submit your next manuscript at $\gg$ springeropen.com 